\title{
Peer-led approaches to dietary change: report of the Food Standards Agency seminar held on 19 July $2006 \dagger$
}

\author{
Sigrid Gibson on behalf of the Food Standards Agency \\ Sig-Nurture Ltd, 11 Woodway, Guildford, Surrey, GU1 2TF, UK
}

Submitted 1 May 2007: Accepted 10 May 2007: First published online 3 August 2007

\begin{abstract}
Objective: To summarise the discussions of a seminar on peer-led approaches to dietary change held at the Food Standards Agency (FSA) on 19 July 2006.

Design: Following presentations on three FSA-commissioned research projects involving peer-led dietary interventions, discussions in small workshop groups identified learning points for researchers, policy-makers and practitioners and considered how the findings of such studies can be effectively communicated to each of these groups. The target groups in the three separate projects were: older people living in sheltered accommodation; mothers and babies during the weaning period; and people with diabetes.

Results: The projects discussed here were quite different from one another, involving three separate populations, having different aims and approaches and different styles of peer leading. Their effectiveness in achieving quantitative dietary change was disappointing. However, results for some of the qualitative outcomes (social, psychological, behavioural) were more positive. Process evaluation, whether built in or post hoc, provided useful learning to inform future projects and potentially improve their effectiveness and usefulness for researchers, policy-makers and health promotion practitioners.

Conclusions: The projects discussed here showed that peer-led interventions can achieve positive changes in outcomes such as knowledge, confidence and attitudes, as well as small improvements in diet. They also demonstrated that there is a need for a more sophisticated analysis of peer-led interventions that recognises the diversity of approaches and their suitability in different situations.
\end{abstract}

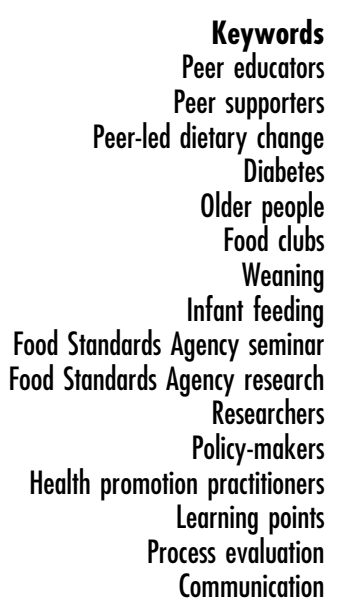

\section{Introduction and aims of the seminar}

The Food Standards Agency's (FSA's) Food Choice research programme aims to identify the social, psychological and physical barriers to achieving a healthier diet and how they might be addressed. Three peer-led intervention studies had been commissioned under research programmes N09 (Food Acceptability \& Choice) and N14 (Food Choice Inequalities). Dr Louis Levy (Head of the Nutrition Policy \& Advice Branch in the Nutrition Division) outlined the scope of these programmes to set the context for the projects that were the focus of the meeting (Fig. 1).

The seminar was chaired by Lynn Stockley (external advisor to the FSA on the Food Choice research programmes). Its aims were to identify the specific and general learning points from the peer-led projects and to discuss how these could be best communicated to various audiences, including practitioners, researchers

tThe views and facts expressed in this report are those expressed by the delegates who attended and spoke at the meeting. and policy-makers. This report summarises each project's findings (both positive and negative) and suggests some learning points to inform future interventions. It also outlines general considerations for researchers, practitioners and policy-makers arising from these intervention studies and suggests some communication routes for disseminating FSA research to various stakeholders.

\section{Promoting recommended infant feeding practices in a low-income sample (N09016)}

Professor Richard Watt (Professor of Dental Public Health, Department of Epidemiology and Public Health, University College London) described the aims of this intervention as being to help fill the evidence gap on how to support mothers of young babies in relation to weaning practices.

\section{Study aims and metbods}

The aim of the study was to design, implement and evaluate a peer support intervention to promote recommended infant feeding practices between the ages of 3 and 12 months. Three hundred and twelve mothers 


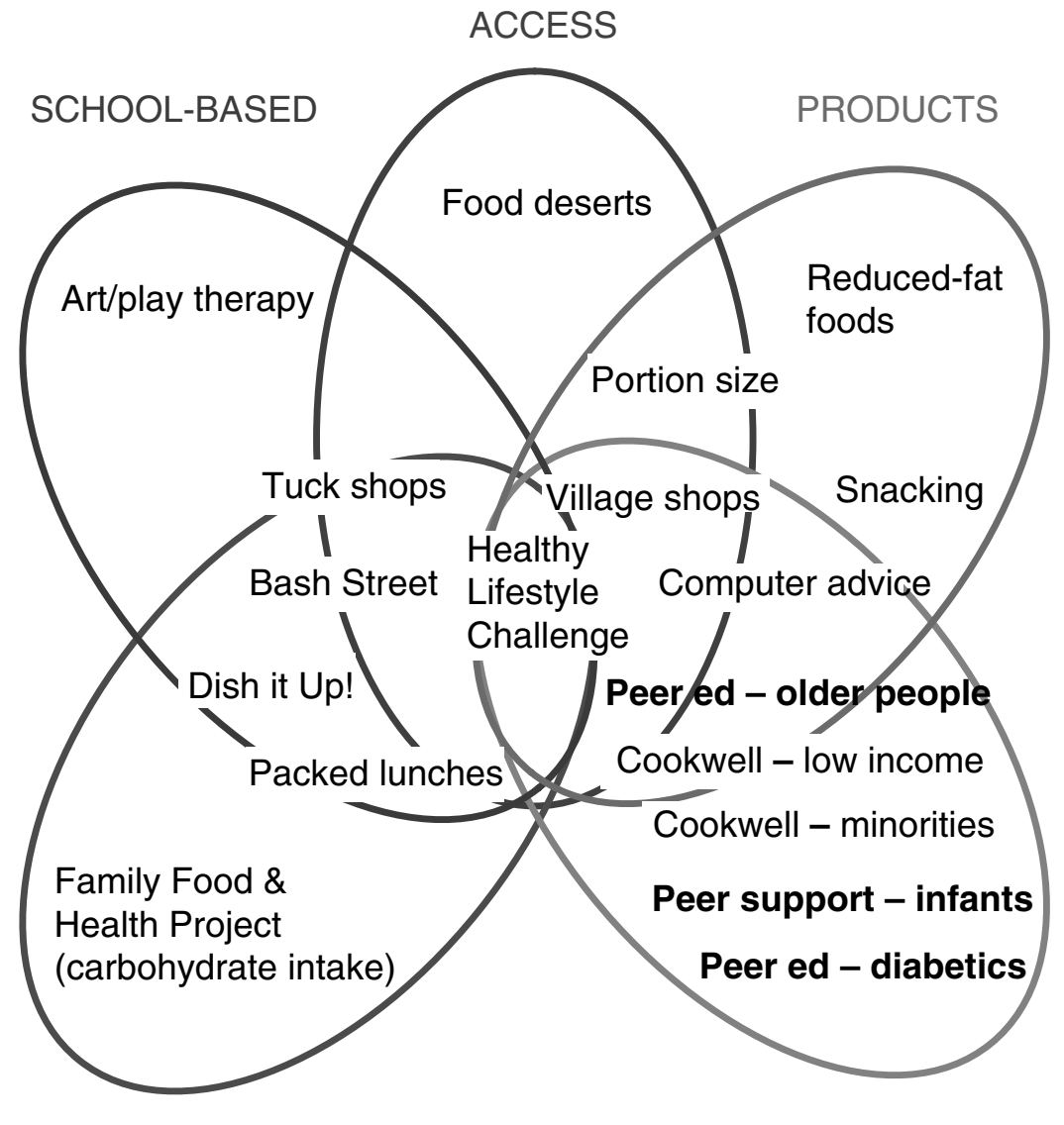

FAMILY-CENTRED

COMMUNITY-BASED

Fig. 1 Overview of research areas commissioned under the Food Acceptability \& Choice and Food Choice Inequalities research programmes

were recruited at baby clinics in deprived areas of Camden and Islington and allocated at random to intervention (157) or control group (155). A group of local volunteers were recruited and trained to provide non-judgemental support and practical assistance on infant feeding, and in particular weaning practices. Home-based support was offered over a 9-month period (an average of five visits of 60 minutes) until the infants were 12 months old. Mothers in the control group received standard professional care only. Data were collected at baseline (10 weeks old), postintervention (12 months old) and at follow-up (18 months old). Nutritional data were collected using the 24-hour multiple-pass recall method, food-frequency questionnaire (FFQ) and interviews. The primary outcome was vitamin $\mathrm{C}$ in fruit, although other macro- and micronutrient intakes were also assessed. Secondary outcomes were fruit and vegetable consumption, feeding practices, child growth and health, and use of health services. Information was also gathered on the mother's health, well-being, fruit and vegetable consumption, and nutritional knowledge and confidence.

\section{Results}

Response rates were $77 \%$ and $68 \%$ at the 12 - and 18 -month follow-up, respectively. At 12 months and also at 18 months there was no evidence of difference in nutritional outcomes between the intervention and control groups. However, there were some indicators of positive change in weaning practices and introduction of family foods. No differences were found in the duration of breast-feeding, but mothers in the intervention group were less likely to have introduced cows' milk before 6 months and were less likely to be still using a bottle at 18 months. Children in the intervention group were more likely to be eating family foods and to be having three solid meals per day. The FFQ data showed that at 12 months children in the intervention group were more likely to be eating carrots, boiled potatoes, apples and pears daily compared with those in the control group, and at 18 months were more likely to be eating chips, boiled potatoes and pears. There were no significant differences in anthropometric measurements between the groups at 12 months. More children in the intervention group were reported to have skin problems and be prescribed medication than those in the control group. At 18 months' follow-up, no significant differences were found in terms of the children's health, use of health services or medication use. No significant differences were detected in the mothers' health and well-being, or their consumption of fruit and vegetables, at either 12 or 18 months' follow-up. However, at the 18-month follow-up 
mothers in the intervention group were more knowledgeable about when bottle-feeding should be discouraged and were more confident in following health professionals' recommendations on how best to feed their child. Women in the intervention group were very positive about the volunteers' support. Process evaluation also indicated that the volunteers highly rated their experience of being involved in the study.

\section{Conclusions}

Although the study did not demonstrate significant positive nutrition or health outcomes, it achieved modest benefits in infant feeding practices and mothers' knowledge and confidence. The Camden Primary Care Trust (PCT) has now implemented a peer support infant feeding initiative using various resources generated by this project.

\section{Learning points from the infant feeding study}

\section{Study design and metbods}

- The planned process evaluation was a highly useful component of the study protocol and provided evidence of benefit in other 'softer' outcomes (knowledge, confidence, attitudes). This evidence was consistent with the suggestion of more promising dietary habits (such as introduction of family foods) in the intervention group.

- The importance of the initial developmental stage (study design and logistical planning) should not be underestimated. Although not originally planned for, a coordinator of volunteers became vital for project management.

- The main outcome measure (vitamin $\mathrm{C}$ in fruit) may not have been the best choice. Vitamin $\mathrm{C}$ intake was above the recommended levels and it may not have been realistic to expect to raise it much higher. Other nutrients, such as iron, might be more critical in this age group but this was thought to require blood samples for iron status, which would not have been feasible.

- It is difficult to engage the most vulnerable individuals in interventions (the sample group contained more breast-feeding mothers and fewer bottle-feeding mothers than expected).

- The dynamic nature of weaning could not easily be captured by the methods used (telephone call at 6 months, interview at 12 months).

- Problems with the software used for analysing the 24-hour recall necessitated use of a less robust method (FFQ) for the food consumption data.

\section{Recruitment of peer supporters}

- Recruitment of volunteers was more difficult and timeconsuming than envisaged, necessitating four waves of recruitment. Interviews, police checks, safety and health all had to be covered in a detailed fashion as the volunteers were doing unsupervised home visits.

- The most successful way of recruiting the volunteers was by placing adverts in free newspapers.

\section{Training and support}

- The training programme required four waves to fit the extended recruitment schedule and to accommodate the busy lives of the volunteers, who were women with children.

- Training time on nutrition and weaning issues was limited, because other issues (communication skills, personal safety, services available locally, etc.) had to be covered as well.

- A dedicated volunteer coordinator was essential to support volunteers, match them to individuals, monitor systems and pay expenses. This was not included in the original study design but additional funding was provided by the FSA.

- Provision for childcare and travel expenses was essential for these volunteers.

- The celebration event to thank volunteers was greatly appreciated. Accreditation would have been desirable but proved too difficult to arrange.

\section{Sustainability (ongoing community intervention)}

- This programme is now being used in Camden PCT, using resources generated by the project. Consideration should be given to resourcing such outputs for wider use (e.g. production, marketing, dissemination, support).

- To do peer support well requires resources and investment from PCTs and others, and commitment to ongoing recruitment and training as people move on.

- Peer support may be more effective if embedded within a multifaceted approach which aims to address the broader determinants of infant feeding and wider family needs.

\section{Can peer educators influence healthy eating in people with diabetes? (N14001)}

Professor Janet Cade (Nutritional Epidemiology Group, University of Leeds) described the aim of this research project: to develop and evaluate a peer educator-led intervention designed specifically for people with type 2 diabetes.

\section{Study metbods}

The intervention consisted of a self-management programme, the Expert Patients Programme (EPP), used in the National Health Service (NHS). There is some evidence 
that these approaches can improve health in regard to the severity of symptoms, decreasing pain, increased activity and improved life control and satisfaction, but there is no information regarding the effect on eating behaviour. People with type 2 diabetes were recruited from general practices in the Burnley, Pendle and Rossendale area of Lancashire. Five volunteer peer educators (tutors), who were themselves diabetic, were enrolled on the EPP and trained to deliver the EPP to the trial participants. The 6-week EPP covered issues common to any chronic disease, including exercise and relaxation, correct use of medication and communication with health-care providers. Action planning, feedback and support were an important part of the programme. The intervention group received six EPP sessions of two hours each, led by the peer educators, plus one supplementary diabetes-specific session, developed by the University of Leeds, covering common problems and the self-management of diabetes through food intake, activity and goal setting. The control group received standard diabetic care only (15-minute consultation with a dietitian or 30 minutes for newly diagnosed diabetics). Outcome measures were food and nutrient intakes, body weight, body mass index and glycosylated haemoglobin levels (HbA1c), a measure of diabetes control.

\section{Results}

From 1726 subjects targeted as potential participants, 319 consented to take part and baseline measurements were available from $112 \mathrm{EPP}$ and 127 control subjects (total 239 subjects). Due to considerable dropout among the EPP group, data on both baseline and 12 -month clinical measures were available only on 63 (56\%) EPP and 91 (72\%) control participants, with dietary data on 74 (66\%) EPP and 103 (81\%) control participants. The study failed to show any significant difference in outcome between intervention and control subjects at 12 months. Qualitative interviews conducted to explore this issue revealed that participants had been dissatisfied with the lack of diabetesspecific information provided through the EPP (dropout had already occurred before the diabetes-specific module in the seventh session). There was also frustration that questions about diabetes could not be answered by the lay tutors. However, tutors and their trainers were positive about the benefits of the EPP, at least in terms of promoting a sense of well-being and quality of life.

\section{Conclusions}

The intervention did not achieve measurable significant outcomes but has highlighted difficulties in conducting health-care research involving multiple agencies and suggested possibilities for improvement.

\section{Learning points from the diabetes study}

\section{Study design and metbods}

- The patient literature given to patients before entering the study, which emphasised diabetes, raised false expectations about the programme content, which was mainly generic and lay-led.

- Placing the diabetes-specific module at the beginning of the EPP, rather than at the end, might have reduced dropout.

- Difficulties with follow-up at 6 months and 12 months were alleviated by access to general practice records and financial incentives.

- Some outcomes (e.g. reduced systolic blood pressure) may be clinically significant but low sample size led to a non-significant result. Interpretation should look at effect size in pilot studies as well as $P$-value. Worthwhile effects could then be explored in future studies using larger samples.

\section{Recruitment of peer educators (tutors)}

- Recruiting diabetics to this role was extremely difficult, especially in some ethnic groups. The initial aim had been to recruit 30 peer educators but only five were eventually recruited. Illness also caused dropout.

\section{Recruitment of participants (recipients)}

- Initial response rate (approximately 20\%) was consistent with experience from other randomised controlled trials.

- Delays in provision of course materials, translation and the introduction of new requirements on the supervision of EPP delivery led to a high dropout rate between recruitment/randomisation and baseline measurements. These delays were mostly beyond the control of the researchers.

\section{Lessons for future studies}

- Future studies need to determine the best type of peer support for the population, find ways of engaging and rewarding peer leaders, provide complementary professional support for answering participants' questions, and achieve maximal follow up.

- For participants, the shared experience (of tutors being diabetic) was less important than getting answers to their questions. Since tutors were not allowed to answer diabetes-related questions, participants sometimes filled the gap by exchanging advice, some of which may have been incorrect. Staging the diabetes session earlier in the course could have dealt with some queries. Some means of referral could be considered for questions arising later. 
- Results from the nationwide EPP (NHS) are due soon and will provide a valuable comparison for these results.

\section{Design and evaluation of peer-led community-based food clubs (N09015)}

Dr Paula Moynihan (Senior Lecturer, Nutrition, University of Newcastle) described the aims of this project: to develop and evaluate a community-based peer-led food club for older adults from socially deprived backgrounds and to investigate whether a peer-led nutrition intervention is feasible, effective and cost-effective in improving diet and health in older adults.

\section{Study metbods}

A 20-week food club, designed by a home economist and a dietitian, included a recipe file of easy-to-prepare dishes that were low in fat and sugars, high in fruit, vegetables, fibre and vitamin $\mathrm{D}$, and a session plan with nutrition notes. Twenty-one people aged 60 years and over were trained to become community nutrition assistants (CNAs) delivering the food club. The project aimed to recruit 288 older adults across 32 sheltered housing schemes in socially deprived areas of north-east England, half of whom would participate in a food club. Information was collected on diet (using a 3-day food diary), vitamin status, anthropometric measurements, knowledge and attitudes to healthy eating at baseline, immediately after the 20-week food clubs and at 1 year.

\section{Results}

The average age of participants was 76 years, $85 \%$ were women. Thirteen out of 16 schemes completed a food club (97 subjects, 104 controls). Information on diet was collected from 201 subjects at baseline, but from only 94 subjects ( 49 food club, 45 control) post-intervention and 72 subjects ( 36 food club, 40 control) at 1 year. Baseline diet was high in saturated fat but not high in sugars, and low in fibre, fruit and vegetables and vitamin D. Immediately following the intervention, no significant changes in diet were observed (adjusted for baseline). There were small changes at 1 year, including significantly higher carbohydrate intake $(+2.4 \%$ of energy) in the intervention group and a non-significant increase in fruit intake $(25 \mathrm{~g})$. Conversely, a negative finding was that vitamin $\mathrm{D}$ intake had fallen in the intervention group and increased in the control group. At baseline $76 \%$ of subjects were overweight or obese; there was no change following the intervention and no change in energy intake. The food club did not result in any changes in the knowledge of nutrition or food safety. The subjects viewed their diet as more healthy than it actually was ('optimistic bias') and this was increased in those who had attended a food club. Perceived barriers to change included access to supermarkets, inability to shop for oneself, waste (inability to obtain small quantities) and age ('too old to change'). Positive outcomes were that some had regained interest in cooking for themselves. The participants' views of the food club concept and its delivery by peer leaders were very positive, and peer leaders were also positive about their experience and the responsiveness of the participants. The cost to train one peer educator was $£ 700$ and the average cost of running a food club was $\$ 130$ per week, or around $£ 70$ if each peer leader ran five food clubs (based on remuneration at the minimum wage rate).

\section{Conclusions}

The diet of many older adults remains poor and many are overweight. Effective interventions are needed to tackle barriers to healthy eating including poor nutrition knowledge, optimistic bias, social isolation, resistance to change and lack of access to small portions.

\section{Learning points from the study of food clubs for older people}

\section{Study design and metbods}

- Dietary programmes designed around quick, one-pot meals with little or no waste, using an interactive 'handson' approach, were well received.

- A larger sample is required to test effectiveness in terms of dietary change.

- Interventions may have unanticipated negative effects (e.g. decreasing vitamin D intake or increasing optimistic bias, which is a barrier to further change).

- Older adults may find it hard to change dietary habits; interventions using adults closer to retirement age may be more successful.

\section{Recruitment of peer educators}

- It is possible to recruit and train older adults to become peer leaders: training in small groups, paying a wage and working towards a qualification were all positively received.

\section{Recruitment of participants (recipients)}

- Recruitment of subjects was very difficult and took two months longer than expected.

- There was a withdrawal rate of over $70 \%$ compared with the 33\% allowed for. Hence the study became underpowered.

\section{Lessons for future studies and intervention programmes}

- Research is needed into how to overcome the reluctance of older adults to take part in dietary interventions. Studies are also needed to test the acceptance and viability of food clubs for men only (using male CNAs). 
- Effective interventions are needed to address obesity in older adults from lower-income backgrounds (diet and exercise programmes).

- Improve access to small portions of fruit and vegetables (e.g. via shared box schemes) and assess impact on diet and health.

- Address the poor nutrition knowledge and food safety practices of this group.

- Counteract optimistic bias (e.g. by giving individual feedback).

- Allow for long-term follow-up.

\section{Overall learning points and communication strategies}

In summing up the morning's discussions, Lynn Stockley (Chair) highlighted the diverse nature of the three interventions, which could all be described as peer education but were in practice quite different in their target group of recipients, in their training, support and remuneration of peer leaders, and in the style of sessions. The attributes of peer educators and their recruitment, training and commitment may all influence the success of such interventions and different approaches (e.g. one-toone or group sessions, didactic or non-judgemental styles) may be effective in different population groups. An approach which is successful in one setting may not translate to a different one. Thus, in evaluating the effectiveness of peer education, there is a need to become more sophisticated in thinking about the context and avoiding generalisations.

The afternoon sessions were devoted to drawing out some more general lessons from the three projects, as applicable to three groups of people: researchers, practitioners and policy-makers. Delegates were also invited to suggest how these lessons might be communicated.

\section{Introduction}

Researchers, practitioners and policy-makers have different aims and this must be recognised in evaluating the effectiveness of projects and lessons learned. The researcher aims to test a hypothesis using predetermined methods and outcomes and follows a protocol that is rigorous but may also be narrow and inflexible. However, many of the issues relevant to researchers are also relevant to practitioners and policy-makers. Practitioners aim to implement an intervention to address the needs of the group, which may be multifaceted and involve a more holistic approach. Policy-makers are interested in the effectiveness of a specific intervention, value for money, how it informs policy and its impact on other sectors. It should also be recognised that these groups are not homogeneous but represent different disciplines, roles and responsibilities. Finally, participants should not be ignored as they too have individual aims such as better health and well-being, emotional and social support, and, in the case of peer leaders, knowledge, skills development and potentially employment.

\section{Learning points for researchers}

Study design

- Consultation with community leaders, other professional disciplines and researchers with relevant experience is important from the planning stage onwards. Research studies based in the community have to be realistic and viable. Is the planned intervention likely to work in that group within the timescale chosen? Do the participants need to change their diet/behaviour and are they motivated to do so? Consider doing a feasibility or pilot study.

- Selection of outcomes is vital. What are the reasons for choosing the main outcome variable? Are there secondary outcomes and is the study sufficiently powered for these? Power calculations may need to assume a pessimistic scenario with $30-50 \%$ dropout, depending on the population and method.

- Are the measurement methods for each outcome both appropriate and accurate? The results suggest that more sensitive assessments for social outcomes need to be sought or developed.

- Consider whether a peer-led approach is appropriate for this intervention and population, or whether a professional input might be more effective and costeffective. If a peer-led approach is taken, this is not a substitute for integration with professionals. Multifaceted 'joined-up' approaches work best, although coordination requires more effort.

- Build in a process evaluation. If the intervention is disappointing, it helps to know why, what did work and what could be done differently to improve it.

\section{Recruitment, training and support}

- Recruitment and retention in community situations is much more difficult than in academic establishments. Anticipate problems with recruitment and resource appropriately to reduce bias and minimise delays.

- High dropout can jeopardise project success by escalating costs, delaying time schedules, biasing randomisation and leading to null results. Essentially these studies became pilot studies with non-significant outcomes for the scientific hypotheses under test.

- Consider remuneration and/or other incentives for peer workers, in order to boost recruitment and retention. Incentives may also be appropriate for participants. Ethical and legal advice is required.

- Training of peer educators needs to be specific, at the correct level, ongoing and responsive. Training should involve professionals from appropriate disciplines. 
Some form of quality assurance is important in the training of peer leaders and in the delivery of the intervention to recipients.

\section{Learning points for practitioners}

- Intervention programmes have more freedom than research studies to tailor projects to the needs of individual recipients, and monitor effectiveness on an individual basis.

- Check expectations. Find out if recipients are more concerned about the competence of educators to answer questions than whether they are peers or fellow patients.

- Existing community volunteer networks/charities/faith groups should be involved and may provide insights, access to participants and peer leaders.

- Incentives that are appropriate to the participants and peer leaders need to be found. These may be monetary or non-monetary (e.g. accreditation).

- Appropriate training resources may already exist and have been evaluated (e.g. EPP) or could be adapted from other projects. All the projects reviewed in the seminar have generated resources that could be utilised.

- If resources permit, consider developing a toolkit from the intervention experience and resources generated.

- Training has a knock-on effect in the lives of peer leaders and their families and may be a springboard to other opportunities for employment or volunteering. This secondary benefit is not easy to quantify.

\section{Learning points for policy-makers}

- The success or otherwise of peer-led interventions depends in part on how they are presented and the expectations of the recipients. The interventions might have been more successful if they had started from the basis of a needs assessment of the target group, and developed the content and delivery around that.

- Funding agencies and researchers need to maintain a constant dialogue and be flexible to changes in protocol that may become necessary to ensure success of the project.

- It is necessary to have a pessimistic expectation of recruitment and dropout in community projects. A contingency plan to mitigate possible failures should be discussed with contractors. Pilot studies may be advisable.

- Evidence is needed of successful approaches to enhance participation in research, surveys and interventions. This has implications across all sectors, not just for the FSA.

- Ethics committee constraints (e.g. confidentiality, opt-in vs. opt-out) may be hampering research. Debate (including public debate) on this is required.
- Social scientists argue that other approaches may provide useful data at less cost and with fewer practical difficulties than randomised controlled trials. However, many believe such trials are needed to assess the effect of interventions in an unbiased way. Discussion may be warranted of appropriate research methods and how difficulties can be overcome.

- More information or links to relevant projects and intervention experience could be given by the FSA in research application guidance documents.

- Short-term projects are resource-intensive to set up. Policy-makers should have an eye to sustainability and ongoing funding and follow-up, where there is evidence of benefit.

- Resources generated by projects (including training materials, data, samples) could be utilised further in consultation with contractors and other professionals.

- Further research on the relative effectiveness and cost of lay (peer) vs. professional support in different populations may be required. These three projects do not assess whether peer-led approaches are better than professional-led or whether one style is better than another (didactic/educator vs. non-judgemental/supporter).

\section{Communication}

The consensus from the workshop's discussion was that communication is a shared responsibility between researchers and funding agencies. The ubiquity of most methods of communication means that the various groups can all be reached by most methods, although some may have greater impact for certain groups. Suggested methods are outlined below but were not discussed in great detail. The content, style and methods of communicating project results and conclusions may be worthy of a seminar in its own right.

\section{General communication methods}

- Final reports of all FSA-commissioned work are archived in the FSA library and information centre and brief lay summaries are featured on the FSA website (www.food.gov.uk) and in the FSA News publication. However, access and timing is an issue as full reports are only made available some months after the project and mostly as hard copy (not downloadable).

- Reports of FSA seminars and workshops on a given theme are a further means of communicating project results and their implications for different stakeholders. Results of several meetings have been successfully disseminated via the FSA website and by publication in journals.

- Using the internet is a highly efficient and cost-effective means of communicating results and is used extensively by all groups. The FSA website is an obvious home for results but there may be difficulties regarding the 
amount of material to be stored and the need for updating or turnover. A first step could be construction of a database of various types of outputs from projects with links to further information or resources.

- Websites of other providers and agencies (e.g. NHS, Diabetes UK, British Dietetic Association) could also be used to disseminate results to practitioners and researchers. For example, the diabetes project discussed at this seminar is linked to the EPP and results will be communicated through this network (website and newsletter) and through Diabetes UK. The EPP could be used as conduit for programmes to be extended into other areas of the UK where funding permits. Given the relevance of these FSA projects for the health, welfare and community services, the question of who is responsible for this communication and what is the best mechanism needs to be explored further. The Department of Health has a role to play in liaising with the FSA on communication of research results and materials relevant to PCTs and the NHS health trainers. Use could also be made of the health libraries network for PCTs, Sure Start and other groups.

- E-mails were discussed as a preferred means of network communication. FSA e-alerts could be used to flag up completed research projects. Professional bodies (e.g. British Dietetic Association, Health Visitors Association) could also be approached to disseminate relevant press releases. Researchers also have their own networks which could be used to cascade information.

\section{Researchers}

- Researchers need to publish research papers in peerreviewed journals for their institution's research assessment exercise and their own career progression. This is important for policy-makers too as the science base becomes a resource for expert committees whose advice informs policy decisions. One problem pertinent to these three projects is that dietary and clinical outcomes were mostly null and hence more difficult to get published. However, it is important that all outcomes both positive and negative are represented or else interventions are viewed through a lens of optimistic bias. By contrast, there was evidence of social benefit and valuable lessons learned in process management that should be disseminated via appropriate journals relevant to these disciplines.

- Researchers are not homogeneous, some work in large institutions while others might be freelance or work in small voluntary-sector organisations. However, most can access professional organisations and multidisciplinary cross-organisational networks such as CHAIN (http://chain.ulcc.ac.uk/chain/index.html). Presentations and seminars on a particular topic may be effective for researchers and others interested in the science and practice of interventions. Science writers could be asked to cascade this more widely to less specialised audiences.

\section{Practitioners}

- Practitioners obtain information from journals, voluntary networks, newsletters, websites, the NHS, the UK Public Health Association, and funding bodies. Articles published in practitioner journals may be a particularly effective means of disseminating results to practitioners in appropriate disciplines. Practitioners need to know all of the results, whether they are beneficial or disappointing. Intervention is as much about the ethos, the approach and the experience of the people involved. Some of this (but not all) can be captured in papers describing the process.

- Cascades could be used to good effect, using a group of individuals to contact people in their area. This is not normally within the control of the Agency and largely depends on the good will and initiative of others. However, the value of liaising with charities and churches could be explored further.

- The materials and training manuals developed in the course of these projects could be publicised to practitioners and utilised further. For example, the FSA has put recipes on the website and plans to add nutritional composition data so these can be used by practitioners running cooking groups. A similar approach could be taken to materials from the projects discussed in this seminar.

\section{Policy-makers}

- Given that policy-makers are a disparate group with different roles, the information disseminated to them must be relevant and have a clear purpose. For example, is it giving research recommendations or relaying evidence endorsing or challenging a policy? Does it have implications for other policy areas or other departments/agencies?

- Policy-makers who are research funders need to consider a communications strategy for their projects from the start and make this an important aspect of the application and discussion with contractors.

- An issue relating to the responsibilities of policy-makers was also discussed. Could or should the FSA be communicating projects and findings wider than currently to avoid duplication with other funders such as regional offices, public health observatories, or even organisations like the Lottery who spend public money?

\section{Conclusions}

There is a need for a more sophisticated analysis of peerled interventions that recognises the diversity of 
approaches and their suitability in different situations. The projects discussed in this seminar were quite different: involving three separate populations and having different aims and approaches and different styles of peer leading. Their effectiveness, in terms of dietary change of the quantitative measures selected, was disappointing, due in part to high rates of dropout that compromised statistical power. Thus the three studies essentially became pilot studies that would require extension to demonstrate significant nutritional and clinical effects. On the other hand, results on some of the softer outcomes (social, psychological, behavioural) were more positive. Most useful of all was the process evaluation, whether built in or post boc, which has provided insights to inform future projects and potentially improve their effectiveness. 